\title{
The exponential behavior of 3D stochastic primitive equations driven by fractional noise
}

\author{
Lidan Wang ${ }^{1}$, Guoli Zhou ${ }^{2}$, and Boling Guo ${ }^{3}$ \\ ${ }^{1}$ Nankai University \\ ${ }^{2}$ Chongqing University \\ ${ }^{3}$ Institute of Applied Physics and Computational Mathematics
}

May 23, 2020

\begin{abstract}
In this article, we study the exponential behavior of 3D stochastic primitive equations driven by fractional noise. Since fractional Brownian motion is essentially different from Brownian motion, the standard method via classic stochastic analysis tools is not available. Here, we develop a method which is close to the method from dynamic system to show that the weak solutions to 3D stochastic primitive equations driven by fractional noise converge exponentially to the unique stationary solution of primitive equations. This method may be applied to other stochastic hydrodynamic equations and other noises including Brownian motion and Lévy noise.
\end{abstract}

\section{Hosted file}

0413-stability-3D-SPE-ver6.pdf available at https://authorea.com/users/325535/articles/ 453526-the-exponential-behavior-of-3d-stochastic-primitive-equations-driven-byfractional-noise 